\title{
Immunological insights on influenza infection and vaccination during immune checkpoint blockade in cancer patients
}

\author{
Melissa Bersanelli*,1,2(D), Stefania Scala ${ }^{3}$, Paola Affanni ${ }^{2}$, Licia Veronesi ${ }^{2}$, Maria Eugenia \\ Colucci $^{2}$, Giuseppe Luigi Banna ${ }^{4}$ (D) Alessio Cortellini ${ }^{5,6}$ \& Francesco Liotta ${ }^{7}$ \\ ${ }^{1}$ University Hospital of Parma, Medical Oncology Unit, Parma, Italy \\ ${ }^{2}$ University of Parma, Medicine \& Surgery Department, Parma, Italy \\ ${ }^{3}$ Functional Genomics, Istituto Nazionale Tumori "Fondazione G. Pascale" IRCCS, Naples, Italy \\ ${ }^{4}$ United Lincolnshire Hospital NHS Trust, Lincoln, UK \\ ${ }^{5}$ Medical Oncology Unit, St Salvatore Hospital, Medical Oncology Unit, L'Aquila, Italy \\ ${ }^{6}$ St. Salvatore Hospital, University of L'Aquila, Department of Biotechnological \& Applied Clinical Sciences, L'Aquila, Italy \\ ${ }^{7}$ Department of Experimental \& Clinical Medicine, University of Florence, Florence, Italy \\ *Author for correspondence: Tel.: +39 0521702 316; bersamel@libero.it
}

First draft submitted: 8 November 2019; Accepted for publication: 20 January 2020; Published online: 12 February 2020

Keywords: cancer patients $\bullet$ flu vaccine $\bullet$ immunological response $\bullet$ influenza vaccination $\bullet$ influenza virus $\bullet$ INVIDla study

\section{Seasonal influenza \& vaccination in cancer patients}

Seasonal influenza affects every country, community and, consequently, a large number of individuals [1]. Recent estimates have recalculated the burden of global influenza. Each year, one billion people are affected by influenza, with a high rate of hospitalization. Deaths associated with respiratory disease from influenza are between 290,000 and 645,000 . Cardiovascular disease deaths, which are estimated to represent about half of deaths due to the complications of influenza, must also be considered [2].

From a public health point of view, the adult or elderly cancer patient presents the same comorbidities as the general population, which increases with age. It is estimated that more than $20 \%$ of the population aged 65 and over have at least two comorbidities, a percentage that reaches more than $50 \%$ in people aged at least 75 years of age [3]. Patients with malignancies are at increased risk of serious influenza-related complications, with higher rates of hospitalization and mortality than healthy cohorts. In several studies conducted during the 2009-2010 influenza pandemic, high rates of hospitalization, pneumonia and death (9.5\%) were reported among oncological patients [4].

Although annual vaccination against influenza infection is recommended, vaccination rates among cancer patients are apparently low [5]. Few patients with a diagnosis of a malignant disease are advised about influenza vaccination by their treating oncologist. The main reasons for influenza vaccination denial are concerns about interaction with the malignant disease, with cancer treatment and potential side effects [6].

A recent Cochrane revision reported lower mortality and infection-related outcomes with influenza vaccination, with limited value to support the benefit of vaccination in cancer patients, due to the small number of studies. According to the current evidence, it seems that the benefits of vaccinating adults with cancer against influenza outweigh the potential risks. However, additional placebo or no treatment arms in randomized controlled trials of influenza vaccination among adults with cancer are ethically questionable [7].

In addition to traditional vaccines produced in embryonated hen's eggs, innovative vaccines are currently available, such as the vaccine produced using a seed virus propagated exclusively in cell culture, the recombinant influenza vaccine manufactured in insect cells and the inactivated mammalian cell-grown vaccine [8]. Manufacturing influenza virus vaccines using a mammalian cell line rather than embryonated chicken eggs may carry certain advantages. First, they overcome the difficulty of growing influenza viruses, in particular the subtype A/H3N2, in embryonated hen's eggs. Second, they help avoid adaptive mutations of the virus in the course of vaccine production. Finally, they 
accelerate timelines for vaccine composition to keep up with the pace of rapid wild virus evolution and respond to potential future pandemics [8]. The potential benefits deriving from these new products could be translated into a more effective influenza vaccination. Moreover, such new techniques will provide basic knowledge for further studies on host-related immune factors and new vaccine formulations (higher doses, use of cell-based or recombinant systems for a single antigen, etc.) and may also help to develop more targeted vaccination strategies against the groups at greatest risk, such as cancer patients.

\section{Cancer patients undergoing immune checkpoint blockade: a special population}

As we previously reported in a systematic review, there are no solid data supporting the efficacy of influenza vaccination in the subgroup of cancer patients receiving immune checkpoint inhibitors (ICIs) [9]. Despite some evidence of good seroconversion rates following influenza vaccination during ICI, which were higher when compared with both those of cancer patients receiving cytotoxic chemotherapy and even of healthy subjects [10,11], prospective data about safety, clinical efficacy and possible interactions of the flu vaccine with anticancer therapy is still largely unknown. In our opinion, serological efficacy is insufficient to support the routine administration of annual influenza vaccination for ICI-treated patients. Looking for explorative data, our group conducted the INVIDIa study, which was the first retrospective study reporting results about clinical efficacy and compatibility of influenza vaccination with ICI therapy $[12,13]$. Soon after, other similar retrospective analyses $[14,15]$ and limited prospective experiences $[16,17]$ were reported in the literature. Considering these studies all together, the evidence related to influenza vaccination during ICI therapy in cancer patients is still scarce and controversial and there is a need for more robust data about safety and efficacy in this population.

Given this unmet clinical need, a multicenter observational trial (the INVIDIa-2 study) is currently collecting prospective data during the 2019-2020 flu season at more than 80 oncology centers in Italy, aiming to provide for recommendations on influenza vaccination in cancer patients treated with ICIs. The INVIDIa-BIOS ancillary study will also investigate, in a large INVIDIa-2 patient subgroup, the immunological background of the immune response to vaccination during ICI, as well as the immune response to ICI during vaccination, with the aim of identifying unexplored immunological interactions.

\section{Immunological insights}

The immunological balance during immune checkpoint blockade

The immune system is a complex network of cells and soluble mediators dedicated to the maintenance of organism integrity. This means not only defending the body from the attacks of external pathogens, but also from possible internal sources of danger (i.e., tumors). This is also known as 'immune surveillance' and it is fundamental for the elimination of cancerous cells before they can grow and give rise to tumor masses. Immune surveillance can be impaired due to the action of immune regulatory cells or due to mechanisms induced by the tumor itself and/or cells of tumor stroma, leading to tumor growth [18-20]. The T-cell response is regulated by a balance between stimulatory and inhibitory signals, called 'immune checkpoints'. Under normal physiological conditions, immune checkpoints are crucial for the maintenance of self-tolerance but also for the protection of tissues from immunemediated damage when the immune system is responding to exogenous antigens with excessive intensity and/or duration. These immune checkpoints are also involved in the impairment of immunity against tumors, thus have been used as targets for antitumor immune therapy. Treatment with ICIs removes inhibitions in the way between the recognition of tumor antigens and the killing of cancerous cells. The blockade of a general immune-regulatory mechanism not only favors the effector phase of the immune-response against cancer, but in general drives the balance between immune regulation and immune activation in favor of activation. This altered balance can lead the immune response toward autoimmunity, known to be one of the major concerns of ICI treatment.

\section{Influenza vaccination/infection in patients undergoing immune checkpoint inhibitor therapy}

The immunological balance described above can also result in hyperactivation of a physiological immune response after vaccination during ICI therapy, along with a subsequent immune reaction once the host encounters the wild virus he was vaccinated against. In fact, in normal conditions, the vaccine stimulates both $\mathrm{T}$ and $\mathrm{B}$ cells, providing a protective humoral immunity with poor involvement of the cellular response during the second exposition to the antigen. In the absence of mechanisms related to the regulation of T-cell activation (as seen during ICI therapy), the second encounter of the antigen (i.e., flu infection after flu vaccination) can lead to the generation of T-cell-driven systemic inflammations, which are responsible for the appearance of the clinical symptoms of flu infection in 
vaccinated subjects [21]. This paradoxical immunological phenomenon could underlie the higher rate of influenza infection among vaccinated cancer patients receiving ICI, as reported by the INVIDIa retrospective study [12]. On the other hand, the immune hyperactivation after flu vaccination and/or influenza syndrome in patients treated with ICIs may exert a direct positive effect on antitumor immunity, as suggested by the increase in overall survival in vaccine/virus exposed subjects in the same study ([13], submitted manuscript). In this regard, it is well known how the activation of the immune system against a foreign pathogen may awaken the antitumor immunity via the presence of pathogens associated molecular patterns and pro-inflammatory cytokines. Pathogens associated molecular patterns, pro-inflammatory cytokines and potentially vaccine adjuvants not only activate the response against a pathogen's antigens, but also against a tumor's antigens via a 'bystander' mechanism. The first report of this dates to WB Coley, now considered the initiator of cancer immunotherapy. At the end of 1800, he reported the case of a patient affected by an inoperable osteosarcoma of the neck that completely healed from the tumor after a spontaneous local erysipelas infection. After this observation, he published a case series of ten patients affected by tumor, treated with germs obtained by erysipelas infections, now known to be the Streptococcus pyogenes [22]. In 1974, Zbar and Rapp described how to obtain an antitumor effective Bacillus Calmette-Guérin, an attenuated strain of Mycobacterium bovis, which then resulted in the effective intravesical treatment of nonmuscle invasive bladder cancer [23]. Nowadays, the identification of a safe and effective immune adjuvant for the stimulation of antitumor immunity is a primary aim of the immunology research. A fundamental aspect of the bacterial-mediated antitumor therapy is the administration of the microorganism/adjuvant at the tumor site, in order to activate APCs and other cells of the immune system at the tumor level and in draining lymph nodes. This phenomenon does not occur during vaccination but may occur during wild flu infection in lung cancer patients. In these patients, the ongoing treatment with ICIs, which is responsible for an increased immune/inflammatory response, could induce the activation of intratumoral APCs via a systemic release of cytokines induced by vaccination and/or by spontaneous viral infection. This could be one of the biologic mechanisms underlying the clinical observation reported within the INVIDIa study $[12,13]$.

\section{Focus on the immunological context}

The tumor microenvironment (TME) is comprised of innate and adaptive immune cells. Although a predominant role was reported for adaptive immune cells, with Th1 cells promoting a pro-inflammatory status versus Th2 cells orchestrating an immunosuppressive phenotype, the innate immune response contributes to the anticancer immunity. Natural killer (NK) cells are circulatory, innate lymphoid cells recognized for their cytotoxic effector functions. Generally, there are two subsets of NKs: CD56hiCD16, which secrete inflammatory cytokines and CD56loCD16hi, which are specialized in cytotoxic functions, along with cell-mediated killing [24]. Within the cancer framework, these cells are extremely efficient in eliminating malignant cells and limiting tumor metastases $[24,25]$. Tumors employ many mechanisms to evade destruction by NKs, engaging inhibitory NK receptors and utilizing platelets as a shield to avoid NK detection [25]. Many cytokines commonly present in the TME diminish NK effector functions through reduced cytotoxicity, arresting the proliferation and expansion of $\mathrm{T}$ cells, enhancing their immune-suppressive properties. Another crucial T-cell type that infiltrates the TME is the Treg, which is responsible for maintaining self-tolerance and impairing antitumor immune responses. The function of Tregs includes direct killing of effector $T$ cells, production of immunosuppressive cytokines and downregulation of co-stimulation by APCs [26]. CTLA- 4 and PD- 1 are well characterized immune co-inhibitory receptors that have been successfully targeted to promote and enhance immune responses against cancer. CTLA- 4 and PD-1 are both induced on T cells upon T-cell receptor signaling activation. On a hand, CTLA-4 is upregulated during the initial stage of T-cell activation, competing with CD28 for the ligands CD86 and CD80 expressed on APCs, thus limiting excessive T-cell priming. On the other hand, PD-1, which is induced later, controls previously activated T cells at the effector sites of immune responses, thus its expression is the marker of T-cell exhaustion. CTLA- 4 and PD-1 checkpoints are deregulated in tumor-bearing hosts, characterized by chronic predominating ineffective immune responses, resulting in Treg induction and T-cell exhaustion [27].

In this complex context, vaccine stimulation may create new scenarios through interfering in the immunemediated innate and adaptive responses. The results reported in the INVIDIa study seem to emphasize the role of added, extrinsic immune stimulation in modulating the efficacy of inhibitory molecules targeting immune checkpoints [12,13]. In cancer patients, the immune status is often modified with prevalence of immunosuppression, thus the direct/indirect viral induced response might impact on the immune patient status, resulting in an antiviral response of which anticancer responses will also benefit from. The recent case of a patient with concomitant primary 
cutaneous anaplastic large cell lymphoma and melanoma progressing on anti-PD-1 therapy, who developed Kaposi's varicelliform eruption after receiving the first dose of herpes virus-based oncolytic viral therapy, appears to flow in the same direction [28]. The fact that Tregs impair function and boost innate populations such as NK may explain part of this process. Preliminary evidence shows that in renal cancer patients, a high basal percent of functional $\mathrm{NK}$ and increased resistance of effector $\mathrm{T}$ cells to Treg suppression predicted a better clinical response to treatment with the anti-PD-1 antibody nivolumab [29]. In melanoma, Tregs from anti-PD-1 responding patients showed a reduction in Treg suppressive capacity [30]. In patients treated with the anti-CTLA4 blocking antibody ipilimumab, higher baseline levels of circulating Tregs are associated with improved survival [31]. PD-1 blockade increased the resistance of effector $T$ cells to Treg suppression and directly reduced the suppressive function of Tregs in vitro [31,32]. Challenges with flu-vaccine or flu wild viruses could test this status. In addition, the immunological and clinical response to flu infection and/or vaccine could be investigated as a possible biomarker of ICI response, as it is being explored within the prospective INVIDIa-2 and INVIDIa-BIOS studies currently ongoing [33,34].

\section{Conclusion}

Cancer patients undergoing immune checkpoint blockade represents a new population still to be investigated from the immunological viewpoint. The complex modifications occurring in the immune responses of these individuals against noncancer antigens are largely unknown and they potentially influence the oncological outcome. The intentional introduction of vaccinal antigens could contribute, as a further exogenous immunological stimulus, to shift the balance between tolerance and activation, likely influencing both the tumor context and the complex relationships between the tumor and the host. Moreover, the effect of exogenous wild stimuli such as viral or bacterial infections may likely contribute to the immunological ensemble, even potentially interfering with the immune escape from antitumor response. In conclusion, the cancer patient treated with ICI can no longer be considered like the entire cancer population undergoing chemotherapy or targeted therapy, since the target of ICI is no longer represented by the tumor, but instead by the immune system [35]. Considering this crucial paradigm, the assumptions adopted up today with regard to concomitant medications, vaccinations, true risks from viral or bacterial infections, should be completely discarded and rewritten for this special cancer patient population.

\section{Acknowledgments}

We gratefully acknowledge all the colleagues from 21 Italian centers who contributed to the first INVIDla study and we are even more grateful to all the colleagues from over 80 Italian centers who are currently contributing to the INVIDIa-2 and INVIDIa-BIOS studies. We also gratefully acknowledge the Federation of Italian Cooperative Oncology Groups (FICOG) for promoting the INVIDIa projects.

\section{Financial \& competing interests disclosure}

The authors are the investigators of the INVIDla study. The authors received research funding from Seqirus, Roche, Novartis, Pfizer, Sanofi, AstraZeneca and BMS. The authors received additional funding from Novartis, Pfizer, Sanofi and BMS for advisory roles along with speaker roles at a scientific event. The authors have no other relevant affiliations or financial involvement with any organization or entity with a financial interest in or financial conflict with the subject matter or materials discussed in the manuscript apart from those disclosed.

No writing assistance was utilized in the production of this manuscript.

\section{References}

1. WHO. Global Influenza strategy 2019-2030. Prevent. Control. Prepare. (2019) https://apps.who.int/iris/bitstream/handle/10665/311184/9789241515320-eng.pdf

2. Iuliano AD, Roguski KM, Chang HH et al. Global Seasonal Influenza-associated Mortality Collaborator Network. Estimates of global seasonal influenza-associated respiratory mortality: a modelling study. Lancet 391(10127), 1285-1300 (2018).

3. Barnett K, Mercer SW, Norbury M, Watt G, Wyke S, Guthrie B. Epidemiology of multimorbidity and implications for healthcare, research, and medical education: a cross-sectional study. Lancet 380(9836), 37-43 (2012).

4. Chemaly RF, Vigil KJ, Saad M et al. A multicenter study of pandemic influenza A (H1N1) infection in patients with solid tumors in 3 countries: early therapy improves outcomes. Cancer 118(18), 4627-4633 (2012).

5. Loulergue P, Mir O, Alexandre J, Ropert S, Goldwasser F, Launay O. Low influenza vaccination rate among patients receiving chemotherapy for cancer. Ann. Oncol. 19(9), 1658 (2008). 
6. Poeppl W, Lagler H, Raderer M et al. Influenza vaccination perception and coverage among patients with malignant disease. Vaccine 33(14), 1682-1687 (2015).

7. Bitterman R, Eliakim-Raz N, Vinograd I et al. Influenza vaccines in immunosuppressed adults with cancer. Cochrane Database Syst. Rev. 2, CD008983 (2018).

8. Barr IG, Donis RO, Katz JM et al. Cell culture-derived influenza vaccines in the severe 2017-2018 epidemic season: a step towards improved influenza vaccine effectiveness. NPJ Vaccines 3, 44 (2018).

9. Bersanelli M, Buti S, De Giorgi U et al. State of the art about influenza vaccination for advanced cancer patients receiving immune checkpoint inhibitors: when common sense is not enough. Crit. Rev. Oncol. Hematol. 139, 87-90 (2019).

10. Läubli H, Balmelli C, Kaufmann L et al. Influenza vaccination of cancer patients during PD-1 blockade induces serological protection but may raise the risk for immune-related adverse events. J. Immunother. Cancer 6(1), 40 (2018).

11. Keam B, Kang CK, Jun KI et al. Immunogenicity of influenza vaccination in patients with cancer receiving immune checkpoint inhibitor. Clin. Infect. Dis. doi: 10.1093/cid/ciz1092 pii, ciz1092 (2019) (Epub ahead of print).

12. Bersanelli M, Giannarelli D, Castrignanò $P$ et al. Influenza vaccine indication during therapy with immune checkpoint inhibitors: a transversal challenge. The INVIDIa study. Immunotherapy 10(14), 1229-1239 (2018).

13. Bersanelli M, Buti S, Banna GL et al. Impact of influenza syndrome and flu vaccine on survival of cancer patients during immunotherapy in the INVIDIa study. Immunotherapy 12(2), 151-159 (2020).

14. Wijn DH, Groeneveld GH, Vollaard AM et al. Influenza vaccination in patients with lung cancer receiving anti-programmed death receptor 1 immunotherapy does not induce immune-related adverse events. Eur. J. Cancer 104, 182e187 (2018).

15. Chong CR, Park VJ, Cohen B, Postow MA, Wolchok JD. Kamboj M safety of inactivated influenza vaccine in cancer patients receiving immune checkpoint inhibitors (ICI). Clin. Infect. Dis. 70(20), 193-199 (2019).

16. Läubli H, Balmelli C, Kaufmann L et al. Influenza vaccination of cancer patients during PD-1 blockade induces serological protection but may raise the risk for immune-related adverse events. J. Immunother. Cancer 6(1), 40 (2018).

17. Gwynn ME, DeRemer DL, Saunders KM, Parikh J, Bollag RJ, Clemmons AB. Immune-mediated adverse events following influenza vaccine in cancer patients receiving immune checkpoint inhibitors. J. Oncol. Pharm. Pract. doi:10.1177/1078155219868758 (2019) (Epub ahead of print).

18. Zou W. Immunosuppressive networks in the tumour environment and their therapeutic relevance. Nat. Rev. Cancer 5(4), 263-274 (2005).

19. Liotta F, Gacci M, Frosali $\mathrm{F}$ et al. Frequency of regulatory $\mathrm{T}$ cells in peripheral blood and in tumour-infiltrating lymphocytes correlates with poor prognosis in renal cell carcinoma. BJU International 107(9), 1500-1506 (2011).

20. Liotta F, Querci V, Mannelli G et al. Mesenchymal stem cells are enriched in head neck squamous cell carcinoma, correlates with tumour size and inhibit T-cell proliferation. Br. J. Cancer 112(4), 745-754 (2015).

21. Hillaire ML, Rimmelzwaan GF, Kreijtz JH. Clearance of influenza virus infections by T cells: risk of collateral damage? Curr. Opin. Virol. 3(4), 430-437 (2013).

22. Coley WB. The Treatment of malignant tumors by repeated innoculations of erysipelas: with a report of ten original cases. Am. J. Med. Sci. 10, 487-511 (1893).

23. Zbar B, Rapp HJ. Immunotherapy of guinea pig cancer with BCG. Cancer 34(Suppl. 4), 1532-1540 (1974).

24. Stabile H, Fionda C, Gismondi A, Santoni A. Role of distinct natural killer cell subsets in anticancer response. Front. Immunol. 8, 293 (2017).

25. Maurer S, Kropp KN, Klein G et al. Platelet-mediated shedding of NKG2D ligands impairs NK cell immune-surveillance of tumor cells. Oncoimmunology 7(2), e1364827, PMID:29308299 (2018)

26. Sakaguchi S, Wing K, Onishi Y, Prieto-Martin P, Yamaguchi T. Regulatory T cells: how do they suppress immune responses? Int. Immunol. 21(10), 1105-1111 (2009).

27. Fife BT, Bluestone JA. Control of peripheral T-cell tolerance and autoimmunity via the CTLA-4 and PD-1 pathways. Immunol. Rev. 224, 166-182 (2008).

28. Miller DM, Trowbridge RM, Desai A, Drews RE. Kaposi's varicelliform eruption in a patient with metastatic melanoma and primary cutaneous anaplastic large cell lymphoma treated with talimogene laherparepvec and nivolumab. J. Immunother. Cancer 6(1), 122 (2018).

29. Santagata S, Trotta AM, Rea G et al. 1906P - Basal NK activity and early Treg function inhibition predicts Nivolumab responsiveness in metastatic renal cancer patients (REVOLUTION) trial. Ann. Oncol. 30(Suppl. 5), mdz268.033 (2019).

30. Woods DM, Ramakrishnan R, Laino AS et al. Decreased suppression and increased phosphorylated STAT3 in regulatory T Cells are associated with benefit from adjuvant PD-1 blockade in resected metastatic melanoma. Clin. Cancer Res. 24(24), 6236-6247 (2018).

31. Martens A, Wistuba-Hamprecht $\mathrm{K}$, Geukes Foppen M et al. Baseline peripheral blood biomarkers associated with clinical outcome of advanced melanoma patients treated with ipilimumab. Clin. Cancer Res. 22(12), 2908-2918 (2016).

32. Wang W, Lau R, Yu D, Zhu W, Korman A, Weber J. PD1 blockade reverses the suppression of melanoma antigen-specific CTL by CD4+ CD25(Hi) regulatory T cells. Int. Immunol. 21(9), 1065-1077 (2009). 
33. Indicazione al Vaccino anti-Influenzale durante Immunoterapia oncologica con inibitori dei checkpoint immunitari. Studio prospettico osservazionale multicentrico:

INVIDIa-2. (2019) https://studiclinici.aiom.it/studi-clinici/ricerca-studi-clinici/indicazione-al-vaccino-anti-influenzale-durante-imm unoterapia-oncologica-con-inibitori-dei-checkpoint-immunitari-studio-prospettico-osservazionale-mul/6-511-13158

34. Sotto-studio ancillare INVIDIa-bios: caratterizzazione immuno-biologica su sangue periferico in pazienti oncologici arruolati nello studio osservazionale INVIDIa-2. (2019) https://studiclinici.aiom.it/studi-clinici/ricerca-studi-clinici/sotto-studio-ancillare-invidia-bios -caratterizzazione-immuno-biologica-su-sangue-periferico-in-pazienti-oncologici-arruolati-nello-studio-osservaziona/6-511-13160

35. Bersanelli M, Buti S. From targeting the tumor to targeting the immune system: transversal challenges in oncology with the inhibition of the PD-1/PD-L1 axis. World J. Clin. Oncol. 8(1), 37-53 (2017). 